Ceci, Exstirpation de la carotis comm. de la r. jugularis et du n. vagus en opérant un carcinoma branchiogène: Sem. méd. No. 43.

Dent, A case of excision of the rectum for carcinoma; death $21 \frac{1}{2}$ years later from secondary deposits in the liver. Lancet. No. 4226, 27. August. p. 596.

Depage u. Mayer, Ueber Ureteren- und Blasenresektion bei ansgedehnten Uteruscarcinomen. Archiv f. klin. Chirurgie. Bd. 74. Heft 1.

Edmunds, A case of carcinoma mammae, treated by oophorectomia. Lancet. No. $42 i 5,11$. Juni.

Fenomenow, Totalexstirpation des lrebsigen Uterus nach Wertheim. Russly Wratsch. No. 33.

Handley, Operationstechnik bei Mammacarcinom. Brit. med. Journ. No. 2283. Jacobsen, A few lessons from fifty cases of operation for carcinoma of the tongue. The Practitioner. Mai.

Nacintosh, Gastroenterostomy for carcinoma of the stomach. Med. Record, 19. März.

Pousson, L'intervention chirurgicale dans le cancer de la prostate. Gazette hebdom. des sciences méd. de Bordeaux 20. März.

Pousson, Cure radicale du cancer de la prostate. Annales des malad. des organes génito-urin. 15. Juni.

\title{
V. Arbeiten, betr. sonstige Heilversuche bei Krebsleiden. Prophylaxe der Krebserkrankungen. Krebsheime.
}

Apolant, Ueber die Rückbildung der Mäusecarcinome unter dem Einfluss der Radiumstrahlen. Deutsche med. Wochenschr. No. 31.

Bisserié, Zwei Fälle von Epitheliom der Zunge mit Radiotherapie behandelt. Bulletin de l'académie de médecine. No. 26.

Imbert, Carcinose prostato-pelvienne diffuse à marche aiguë, guérie par la radiothérapie. Bulletin de l'acad. de médecine. No. 29.

Lery-Dorn, Ein Kankioid auf lupöser Grundlage, behandelt mit Riöntgenstrahlen. Berliner klin. Wochenschr. No. 38.

Pels-Leusden, Ueber Behandlung des Carcinoms mit Röntgenstrahlen. Sitzung der Gesellsch. der Charitéärzte rom 14. Juli.

Perthes, Röntgentherapie des Carcinoms. Archiv f. klin. Chirurgie. Bd. 74. Heft 2.

Werner u. Hirschel, Erfahrungen über die therapeutische Wirlung der Radiumstrahlen. Deutsche med. Wochenschr. No. 42. 